\title{
A Specialized System for Arrhythmia Detection for Basic Research in Cardiology
}

\author{
Michael KOHLHAAS ${ }^{\mathrm{a}}$, Lea SEIDLMAYER ${ }^{\mathrm{b}}$ and Mathias KASPAR ${ }^{\mathrm{c}, 1}$ \\ ${ }^{a}$ Comprehensive Heart Failure Center, University of Würzburg, Würzburg, Germany \\ ${ }^{\mathrm{b}}$ Klinikum Oldenburg, Department of Cardiology, Germany \\ ${ }^{c}$ Department of Health Services Research, Carl von Ossietzky University of Oldenburg
}

\begin{abstract}
The detection of cardiac arrhythmias has a long history in medicine, with current developments focusing on early detection using mobile devices. In basic research, however, the use cases and data differ greatly from the experimental setup. We developed a Python-based system to ease detection and analysis of arrhythmic sections in signals measured on extracted and stimulated cardiac myocytes. Multiple algorithms were integrated into the system, tested and evaluated. The best algorithm resulted in an F1-score of 0.97 and was primarily provided in the application.
\end{abstract}

Keywords. Signal processing, arrhythmia detection, basic research

\section{Introduction}

The detection of cardiac arrhythmia has a long history in medicine [1], starting in 1971 [2]. At present, there is a trend to early detection of arrhythmia using mobile devices such as smartwatches [3]. However, in basic research, use cases, requirements and resulting data differ greatly from the experimental setup and arrhythmia may be induced by substances or treatment types. Systems that ease and automate analysis for specific research questions with graphical user interfaces are not always available, and parts of the analysis tasks often have to be performed manually.

In the present experimental setup, cardiac myocytes (muscle cells) were isolated from mice and measured as single cells, using different substances to study their arrhythmogenic potential. The cells were electrically stimulated with a certain frequency for several minutes according to the experimental protocol. The protocol may vary with the specific research question (for an example, see [4]). When healthy, each stimulation induces a single contraction resulting in a regularly beating myocyte following the pace of electrical stimulation. The contractions were recorded in terms of sarcomere length by light microscopy and fluorescence recordings using an IonOptix setup ${ }^{2}$. The rhythm was continuously monitored. The resulting signals were analyzed manually.

We did not find any commercial nor other software that allows for a simplified analysis of such signals in an easy way. The only other publication that was somewhat comparable was done by Gallett et al. [5], who however analyzed arrhythmias in

\footnotetext{
${ }^{1}$ Corresponding Author, Mathias Kaspar, Department of Health Services Research, Carl von Ossietzky University of Oldenburg, Ammerländer Heerstr. 114-118, 26129 Oldenburg, Germany; E-mail: mathias.kaspar@uol.de.

${ }^{2}$ IonWizard Version 6.6.1.108, IonOptix B.V.; De Boelelaan 1105; 1081 HV; Amsterdam; Netherlands
} 
conscious mice. Thus, the only option for the researcher would be a manual counting or the analysis using statistical tools. However, the former is very time-consuming, and the latter may not provide specific solutions for the research question and, thus, may require programming skills.

Our goal was to provide researchers with a system that simplifies the detection and analysis of arrhythmias in signals recorded on myocytes.

\section{Methods}

\subsection{System design}

We developed an application with a graphical user interface using Python 3.7.6 and Pyforms ${ }^{3}$, illustrated in Figure 1. It allows to load the signal of a single measurement and accompanying stimulation markers. Currently, only a simple tabular data format provided by IonWizard ${ }^{2}$ can be imported, which contains columns for an X (i.e., time) and Y (i.e., sarcomere length in $\mu \mathrm{m}$ ) value, a mark time (i.e., stimulation times) and a mark type (i.e., the mark type of the electric stimulation; this is required since the data may contain marks manually added during experiment execution). After loading a measurement, the data is instantaneously displayed and can be explored using zooming and panning. The graphical user interface allows to detect arrhythmic sections using an algorithm that can be configured with multiple parameters.

In a physiological state, a stimulation triggers a single beat (i.e., contraction). Consequently, the basis for the detection of arrhythmic sections is a detection of beats,

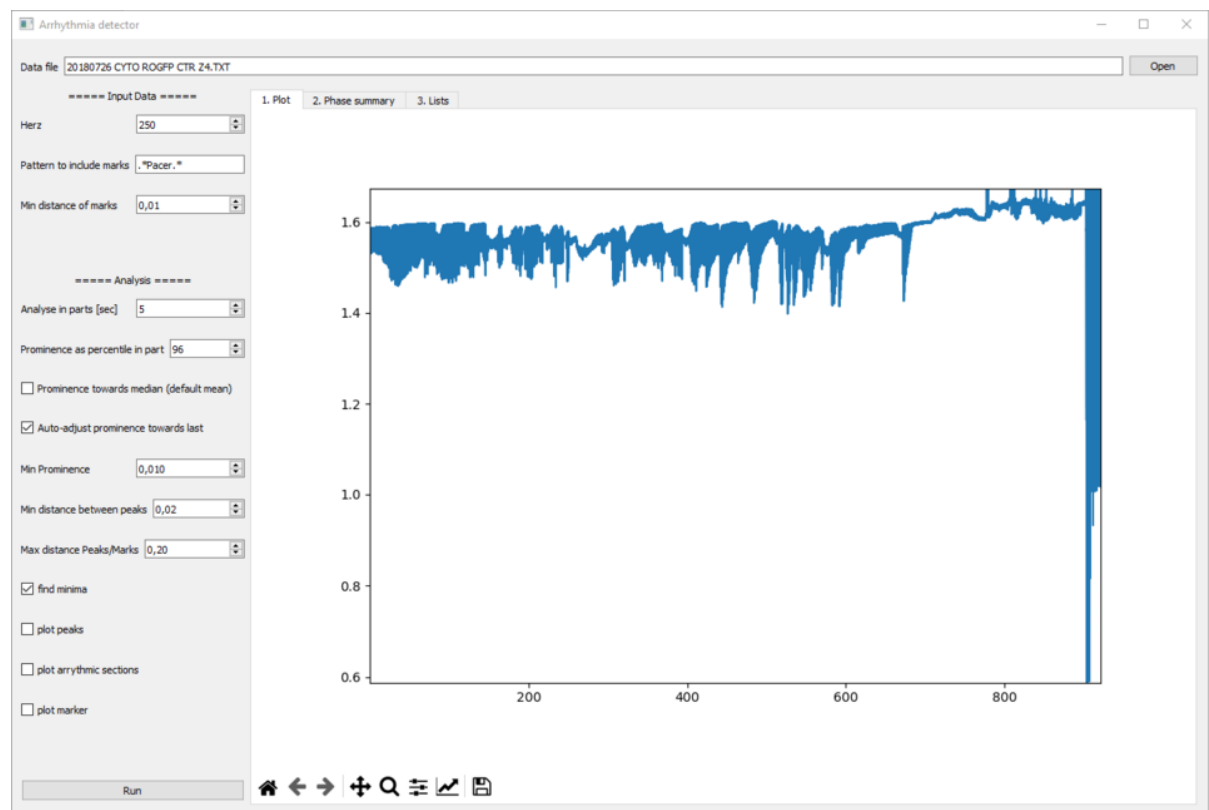

Figure 1. Screenshot of the arrhythmia detector showing the parameter selection on the left and the preview on the right.

${ }^{3}$ Source code: https://github.com/rakmth/myocyte-arrhythmia-detection.git 
which can be reduced to a detection of peaks for this use case. The main difficulty is that beat amplitudes, widths, distances and frequencies vary greatly over time, exposure to a substance, and can be very different for each measurement. After experimenting with different filters on several data sets, we ended up using a simple algorithm that detects peaks without filtering. The whole signal is divided into segments (e.g. $5 \mathrm{sec}$ long, moving windows), in which peaks are detected using the Python SciPy find peaks function and a specific prominence calculated from the segment data (using mean, median, and percentiles) and input from the user interface. The user interface allows to modify multiple parameters: (1) The segment length, (2) the minimal peak prominence (given as percentile of amplitude height versus the mean/median amplitude height), (3) an option to calculate a prominence that is close to the prominence of the previous segment's prominence (several prominence values are calculated for a range of \pm 3 of the given percentile value (cf. variable 2); the prominence closest to the previous one is selected), (4) a minimum absolute prominence, (5) a minimum absolute distance between peaks, and (6) a maximum distance between mark and peak. The manually optimized parameters (cf. section 2.2) are set as application default. Adjustments may be necessary to optimize the peak detection for specific measurements, e.g. including many narrow peaks. After execution, the marks, peaks, and arrhythmic sections may be displayed for review (e.g. as illustrated in Figure 2).

\subsection{Data analysis}

We created a gold standard for arrhythmic sections of signals from 10 cell experiments (overall $>2$ hours of measurement) by a manual visual inspection of the data. Arrhythmic sections start and end at a marker (time of a stimulation). The gold standard was halved in a test and verification set. We used the test data sets to manually optimize the parameters of the moving window algorithm (A1, cf. results in Figure 1) and different static settings for baseline comparison in the peak detection algorithm of the python SciPy library: (A2) a minimum distance of 200 [with $250 \mathrm{~Hz}$, about $90 \%$ of the time between two stimulations], (A3) a threshold of 0.01 , (A4) a prominence of 0.02 , (A5) the setting of (A3) combined with a peak width of 15, and (A6) the setting of (A5) combined with a minimum distance of 40 . Each setting is used statically over the whole measurement.

Evaluation was done with true/false positive/negative values and the metrics precision $(\mathrm{TP} /(\mathrm{TP}+\mathrm{FP}))$, recall $(\mathrm{TP} /(\mathrm{TP}+\mathrm{FN})), \mathrm{F} 1$-score (the harmonic mean of precision and recall, $2 *$ (precision * recall) $/($ precision + recall $)$ ), and balanced accuracy $((\mathrm{TPR}+\mathrm{TNR}) / 2$, where $\mathrm{TPR}=\mathrm{TP} /(\mathrm{TP}+\mathrm{FN})$ and $\mathrm{TNR}=\mathrm{TN} /(\mathrm{TN}+\mathrm{FP}))$. Data presented in the results are based on the verification data set and present macro averages (mean of metric values) of the tested cells.

\section{Results}

A screenshot of the resulting application is shown in Figure 1. A full measurement including identified peaks and sections with arrhythmia as displayed in the application are presented in Figure 2A. Figures 2B-F illustrate details of a normal physiological section (B) and sections with arrhythmia (C-F). Data about identified peaks and sections with arrhythmias can be extracted for further analysis. 
Table 1 presents the macro averages of all metrics and algorithms. The precision is generally high, which means that the algorithm's selections mostly have arrhythmia. However, in the static algorithms, the recall is mostly low, which also leads to low F1scores. The best static algorithms have an F1-score of 0.81 (A2, static distance) and 0.80 (A4, static prominence). In contrast, the moving window algorithm that calculates peaks with adjusted parameters per segment, results in an F1-score of 0.97 . The balanced accuracy also takes true negatives into account and shows similar results.

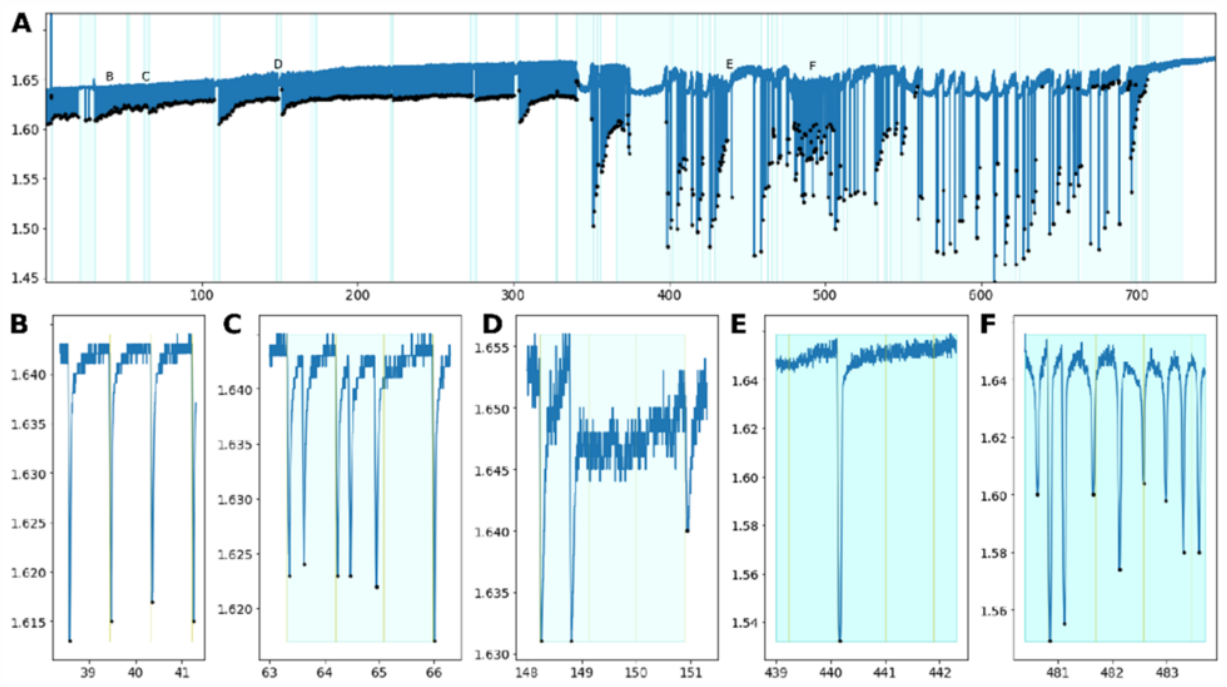

Figure 2. Illustration of an example signal including peaks and arrhythmic sections detected using the moving window peak detection. (A) The full signal with arrhythmic sections being highlighted in light blue. Segments of the signal are magnified in subfigures B-F. (B) A rhythmic section. (C+D) A short arrhythmic section with stimulations resulting in additional and/or omitted beats. (E) A long arrhythmic section except for a single stimulation with beat. (F) Diverse beats without direct relation to the stimulation.

Table 1. Metrics of the moving window and baseline algorithms (c.f. section 2.2): Makro averages of true/false positive/negatives (in percent of all), precision (prec.), recall, F1score, and balanced accuracy (bACC).

\begin{tabular}{|l|c|c|c|c|c|c|c|c|}
\hline Algorithm & TP & TN & FP & FN & Prec. & Recall & F1 & bACC \\
\hline A1. Moving Window & 38.2 & 60.0 & 1.0 & 0.8 & 0.97 & 0.97 & 0.97 & 0.95 \\
\hline A2. Static distance & 36.3 & 47.3 & 2.9 & 13.5 & 0.85 & 0.84 & 0.81 & 0.70 \\
\hline A3. Static threshold & 39.2 & 0.1 & 0.0 & 60.7 & 1.00 & 0.39 & 0.51 & 0.35 \\
\hline A4. Static prominence & 38.2 & 52.5 & 1.0 & 8.2 & 0.99 & 0.73 & 0.80 & 0.81 \\
\hline $\begin{array}{l}\text { A5. Static prominence } \\
\text { and width }\end{array}$ & 38.5 & 34.4 & 0.7 & 26.4 & 0.97 & 0.53 & 0.65 & 0.73 \\
\hline $\begin{array}{l}\text { A6. Static prominence, } \\
\text { width and distance }\end{array}$ & 38.5 & 40.5 & 0.7 & 20.3 & 0.97 & 0.62 & 0.74 & 0.78 \\
\hline
\end{tabular}




\section{Discussion}

We presented an application that supports the identification of arrhythmic sections in basic research measurements on cardiac myocytes. The application allows to analyze single cell measurements using a moving window algorithm that automatically adjusts to quickly changing signals over time.

Since we did not find any other software that allows for such an analysis, we evaluated the system against baseline algorithms typically provided by statistical software. These algorithms have in common that a single defined value was used during the whole measurement. Two of the static baseline algorithms had a decent result. Unexpectedly, the static distance algorithm had the best result of the baseline algorithms. As the stimulation has a constant frequency, using a fixed minimum time will identify each correct beat, but it will also miss any additional beats between stimulations. During the manual training of the algorithms, the best static algorithm we found had a fixed prominence, a fixed peak width and a short minimal distance between peaks, but did not turn out to be the best in the verification. However, all algorithms had difficulties in the peak detection, if there were large signal amplitudes during rest.

The general method allows to determine arrhythmias objectively on the single cell level in order to link them directly to parameters that can only be measured at the single cell level, e.g. cytosolic calcium and sodium concentration. Thus, this method allows to better determine the cellular parameters that have an influence on the development of arrhythmias and thus to develop more targeted therapy options.

\section{Conflict of Interest}

The authors state that they have no conflict of interests.

\section{References}

[1] Luz EJDS, Schwartz WR, Cámara-Chávez G, Menotti D. ECG-based heartbeat classification for arrhythmia detection: A survey. Computer methods and programs in biomedicine. 2016;127:144-164.

[2] Miller AC, Hoare MR, Rey W, Laird JD, Arntzenius AC, Hugenholtz PG. Two approaches to arrhythmia detection by digital computer in the coronary care unit. Folia Med Neerl. 1971;14(5):209-217.

[3] Garabelli P, Stavrakis S, Po S. Smartphone-based arrhythmia monitoring. Curr Opin Cardiol. 2017;32(1):53-57.

[4] Kohlhaas M, Nickel AG, Bergem S, Casadei B, Laufs U, Maack C. Endogenous nitric oxide formation in cardiac myocytes does not control respiration during $\beta$-adrenergic stimulation. J Physiol. 2017; 595(12):3781-3798.

[5] Gallet C, Chapuis B, Oréa V, Scridon A, Barrès C, Chevalier P, Julien C. Automatic atrial arrhythmia detection based on RR interval analysis in conscious rats. Cardiovascular Engineering and Technology. 2013;4(4):535-543. 